\title{
Robotic oligarchy: How a few members can control their whole society by doing almost nothing
}

\author{
Martin Stefanec ${ }^{*}$ \\ Artificial Life Lab of the \\ Department of Zoology \\ Karl-Franzens University \\ Graz, Austria \\ martin.stefanec@uni- \\ graz.at
}

\author{
Gerald Radspieler \\ Artificial Life Lab of the \\ Department of Zoology \\ Karl-Franzens University \\ Graz, Austria \\ gerald.radspieler@uni- \\ graz.at
}

\author{
Martina Szopek \\ Artificial Life Lab of the \\ Department of Zoology \\ Karl-Franzens University \\ Graz, Austria \\ graz.at
}

\author{
Thomas Schmickl \\ Artificial Life Lab of the \\ Department of Zoology \\ Karl-Franzens University \\ Graz, Austria \\ thomas.schmickl@uni- \\ graz.at
}

\begin{abstract}
In swarm systems like honeybees, ants, fish and birds the individual agents show interesting abilities to decide collectively about the swarms behavior based on only locally available information. One example is the BEECLUST algorithm, which is derived from honeybees and was implemented on autonomous robot swarms several times. Here we demonstrate a translation concerning the environmental stimulus: Honeybees aggregate in temperature fields and we used their behaviors to operate robots in a luminescent field to aggregate at the brightest spot. We demonstrate here by a set of experiments that a swarm of 10 e-puck robots is capable to choose a global optimum over a local optimum and we compare those dynamics to real honeybee behaviors. Then we demonstrate that a few "social seed robots" can make the swarm choosing a different option collectively. This allows the swarm to be controlled from the outside without any change of its internal program. We analyze this emergent phenomenon and discuss its implications for future decentralized non-invasive swarm control applications.
\end{abstract}

\section{CCS Concepts}

-General and reference $\rightarrow$ General conference proceedings; •Computing methodologies $\rightarrow$ Multi-agent systems; Artificial life; Mobile agents; Agent / discrete models; Cooperation and coordination;

\footnotetext{
${ }^{*}$ Corresponding author
}

Permission to make digital or hard copies of all or part of this work for personal or classroom use is granted without fee provided that copies are not made or distributed for profit or commercial advantage and that copies bear this notice and the full citation on the first page. To copy otherwise, to republish, to post on servers or to redistribute to lists, requires prior specific permission and/or a fee. BICT 2017, March 15-16, Hoboken, United States

ISBN 978-1-63190-148-5

DOI: $10.4108 /$ eai.22-3-2017.152412

Copyright $\odot 2017$ EAI

\section{Keywords}

collective-decision-making, self-organization, bio-inspired algorithm, honey bees, robot swarm, swarm control

\section{INTRODUCTION}

Groups that make common decisions are ubiquitous in nature on all size and organizational levels: Bacteria [4, 19], social insects [23, 16, 6], birds [18], fish [24] and herding animals [17] are often seen to exhibit collective behaviors in which the emergent collective decision making is driving the behavior of the collective (swarm intelligence) [10]. The collective behavior results from the interactions among individual agents which can be often approximated (when studying natural systems) or programmed (when creating artificial systems) by simple interaction rules.

Similar principles have been found also in human interaction ranging from markets [5], political systems [8], team building [26] and fashions [20]. Also the simple interaction principles can be often identified, e.g. "doing what others do" and "doing something more often, more likely or longer in case it seems beneficial" associated with simple "avoid collisions, conflicts and adverse situations".

Interestingly, a simple laboratory model system was found to show similar properties and rules of interaction: the collective thermotaxis of young honeybees. In a field with different thermal optima, a single young honeybee moves almost randomly around this field, with only a slight preference to warmer areas. Most bees move only randomly through the arena or along the arena wall. Still a single young honeybee is not able to determine a "stable solution" and to locate itself at the thermal optimum. However, a group of bees collectively decides for the optimal temperature spot [22]. Therefore the apparent disadvantage of limited thermoreception of individuals is somehow compensated by the interaction of individuals within the swarm. Further studies showed, that groups of bees are not only able to perform collective thermotaxis and aggregate at the ther- 
mal optimum, but collectively discriminate a local from a global thermal optimum in complex thermal gradients [25]. Detailed analyses revealed that the collective choice of the bees is based on simple local interactions: bees stop when meeting another bee, and the warmer it is the longer they rest [15]. Based on these observations, the BEECLUST algorithm was developed by $[22,21]$. Due to its low requirements, the honeybee-derived algorithm is ideal for implementation on robotic systems. It consists only of four simple rules (An explanatory implementation as pseudo-code is shown in Fig. 2):

1. Move forward. When detecting an object check if it is a wall or another robot (line 00 - line 04).

2. If the object is a wall turn and continue with 1 (line 05, line 06).

3. If the object is another robot measure local illuminance (for bees temperature) and calculate correlated waiting time (line 07 - line 09).

4. When the waiting time is over turn and continue with 1 (line 13 - line 16).

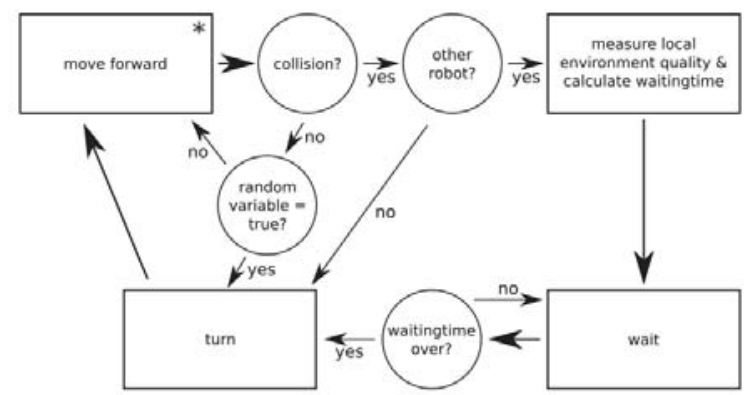

Figure 1: Diagram of the BEECLUST algorithm. The asterisk indicates the starting point of the algorithm. Redrawn from [22].

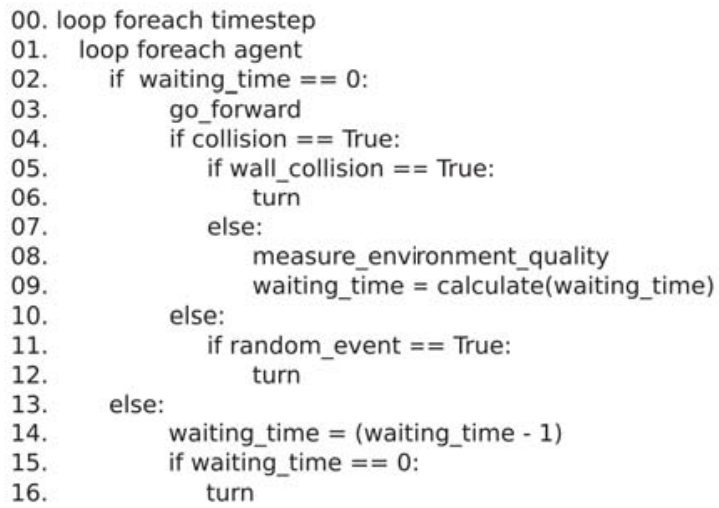

Figure 2: Explanatory implementation of the BEECLUST algorithm as pseudo-code.

With this four simple rules, the BEECLUST is the simplest (so far discovered) swarm algorithm capable of finding the global optimum in a complex landscape of heterogeneous qualities. To accomplish that the individuals do not generate a map of the environment, they do not need self-localization, nor robot-to-robot communication about environmental values. Individual protagonists only relay on random walk and on no other method of orientation, no internal memory is required to store environmental information. In contrast, the swarm itself is a memory that uses the robots and their interactions and locations to build a memory and to compute the environment collectively. The BEECLUST algorithm works even very robustly in a dynamically changing environment [14], all without any centralized control unit.

The simple mechanisms identified in honeybees and expressed by the BEECLUST algorithm can be implemented in robots. The advantage of robots over simulated agents is that they provide the best physics engine available for free: real physics. In order to study the capabilities of such systems in physically embodied agents and compare it to simulated agents, we programmed the rules of BEECLUST into a swarm of robots that has to find a global optimum in a light gradient field containing also a local optimum. The same principles were programmed also into computer agents running in a similar simulated environment.

We focused our experimental work on the scientific question whether or not the presence of a pre-given social seed at the local optimum can drive the swarm as a whole into that final solution, although the same swarm would pick the global optimum without that social seed. And we want to quantize this effect by trying different numbers of social seeds. This set of experiments presented here stands in a line of experiments in which BEECLUST was experimented in dynamically changing light gradients without social seeds [15] and with simulated and robotic agents with one social seed robot in slow-reacting temperature fields $[13,14]$. The study at hand is the first experiment that tests the effect of social seed agents in this system in a light-gradient, which is a fast-reacting stimulus field compared to temperature fields.

Our focal assumption is that a certain number of such social seeds can drive the swarm into unfavorable collective decisions, as they make the swarm to pick the local over the global optimum. Such agents can be seen as malfunctioning agents, or maybe even malevolent agents. However, if such a modulation of swarm control is possible, it offers also a way to control or modulate self-organizing swarms from the outside without switching the general paradigm: The system stays self-organized, decentral, parallel and without a global unit of control. But the experimenter/designer/manager of the system can place environmental incentives to the swarm to alter its behavior.

\section{MATERIAL \& METHODS}

\subsection{Experimental setup}

The here presented robot experiments are primarily based on a previous study where young honeybees were tested concerning their collective decision making abilities in a complex thermal environment (Fig. 7, [25]). Therefore we tried to transfer as many parameters from the real-life honeybee experiments as possible to our robotic experiment setup. While the bees navigated in a temperature gradient with a global and a local thermal optimum (Fig. 5a), the robots were tested in a qualitatively comparable environment with 


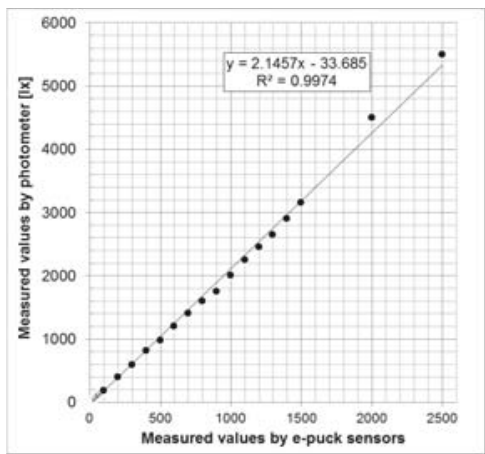

Figure 3: Measured local luminance of the "Artificial Life Lab Color/Brightness Extension Board" compared to the values of the Wetekom ST-2232 photometer

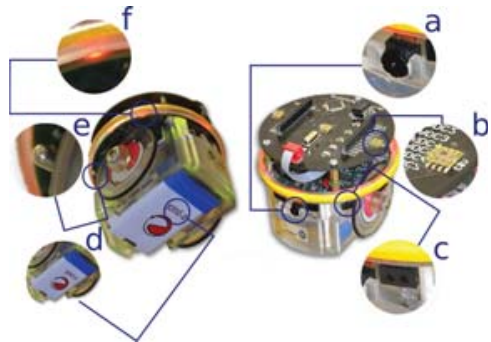

Figure 4: Overview of the e-puck robot with extension board (top):

(a) VGA camera (b) Brightness sensor on top of the "Artificial Life Lab Color Extension Board" (c) Proximity sensor (d) Rechargeable battery (e) On/Off switch (f) LED

a local and a global optimum generated by two light sources (Fig. 5b and Fig. 5c).

We used plastic sheets of different color, dimming the luminance values radiated by the two light sources, to generate a global and a local optimum. One of this sheets being orange (local optimum) and one being transparent (global optimum) to create similar possible distortions (Fig. 6). Overall this leads to a comparable distribution of luminance values, while maintaining a similar size and shape of global and local optimum. The difference in color is not important for the result of the experiments, given that the robots only measured for luminance levels. We targeted luminance values at a maximum of $1000 \mathrm{~lx}$ at the global optimum, $400 \mathrm{~lx}$ at the local optimum and about $0 \mathrm{~lx}$ at the pessimum. We conducted our robotic experiments in a rectangular arena with a length of $200 \mathrm{~cm}$ and a width of $120 \mathrm{~cm}$ $\left(24000 \mathrm{~cm}^{2}\right)$. As the e-puck robot is not capable of measuring light intensities from above, we designed the "Artificial Life Lab Color/Brightness Extension Board", light intensity measurements were performed by a TCS3200 color light-tofrequency converter (whereas we only used it for light intensity measurement). We executed a light intensity calibration of this extension board by correlating it to measurements of a "Wetekom ST-2232" photometer (Fig. 3).

\subsection{Experiments}

Using the BEECLUST algorithm as a controller for our e-puck robots, two sets of experiments were conducted:

- In the first scenario we replicated the above mentioned choice experiments conducted with real honeybees [25]. Swarms of 10 robots were tested in a complex environment with an local and a global optimum $(\mathrm{N}=$ 10 repetitions). We determined the distribution of all robots in the different evaluation zones (global optimum, $11.2 \%$ of the total area , local optimum, $11.2 \%$ of the total area and pessimum, outside the other zones, $77.6 \%)$ at the end of each run (10 minutes).

- In the second scenario we again tested swarms of 10 robots in the same setup, but introduced social seed agents. These are robots that behave essentially equal to "ordinary" robots. Other robots perceive them not as an obstacle, but as a real counterpart and react to it accordingly to the rules of the BEECLUST algorithm. The difference to all the other "ordinary" robots is, that social seed agents are immobilized and can be placed at certain points in the arena, where they will stay for the whole duration of an experiment. We placed two extra robots in the local and the global optimum each (4 additional robots in total). Depending on the experiment, either none, one, or both of these robots acted as social seed agents in the local optimum. Two additional robots were used as dummy robots in the global optimum. The dummy robots in the global optimum can only be perceived as obstacles by the ordinary robots and were used to prevent any unwanted biases in the setup. Again, the distribution of the robots in the different evaluation zones was determined at the end of each run (10 minutes).

To show that our robots, controlled by the BEECLUST algorithm, exhibit comparable collective behaviors to real life honeybees, we determined the number of bees or robots at the end of each run in each evaluation zones. In contrast to honeybees, robots also allow us to monitor their internal states over the whole run time. Hence we were able to determine the number of robots in active waiting mode and analyzed the temporal distribution of robots in waiting mode over the total run time of all experiments. The build-in LEDs of the robot (Fig. 4f) were used to indicate the internal state of each robot. We detected the number of robots in active waiting mode at the global and the local optimum every two seconds over the course of each run (10 minutes). The 'Uniform random distribution Model' (UDM) is based on the assumption that the individual agent (robot or bee) does not take into account neither the other agents nor the temperature gradient. The predicted distribution of agents to the 3 different evaluation zones (global optimum, pessimism and local optimum) therefore corresponds to the size of these zones $(11.2 \%$ for the global optimum and the local optimum, respectively, and $77.6 \%$ for the pessimum area). Deviations in the observed distribution of agents from the UDM predictions indicate that agents locations are not chosen independently. 

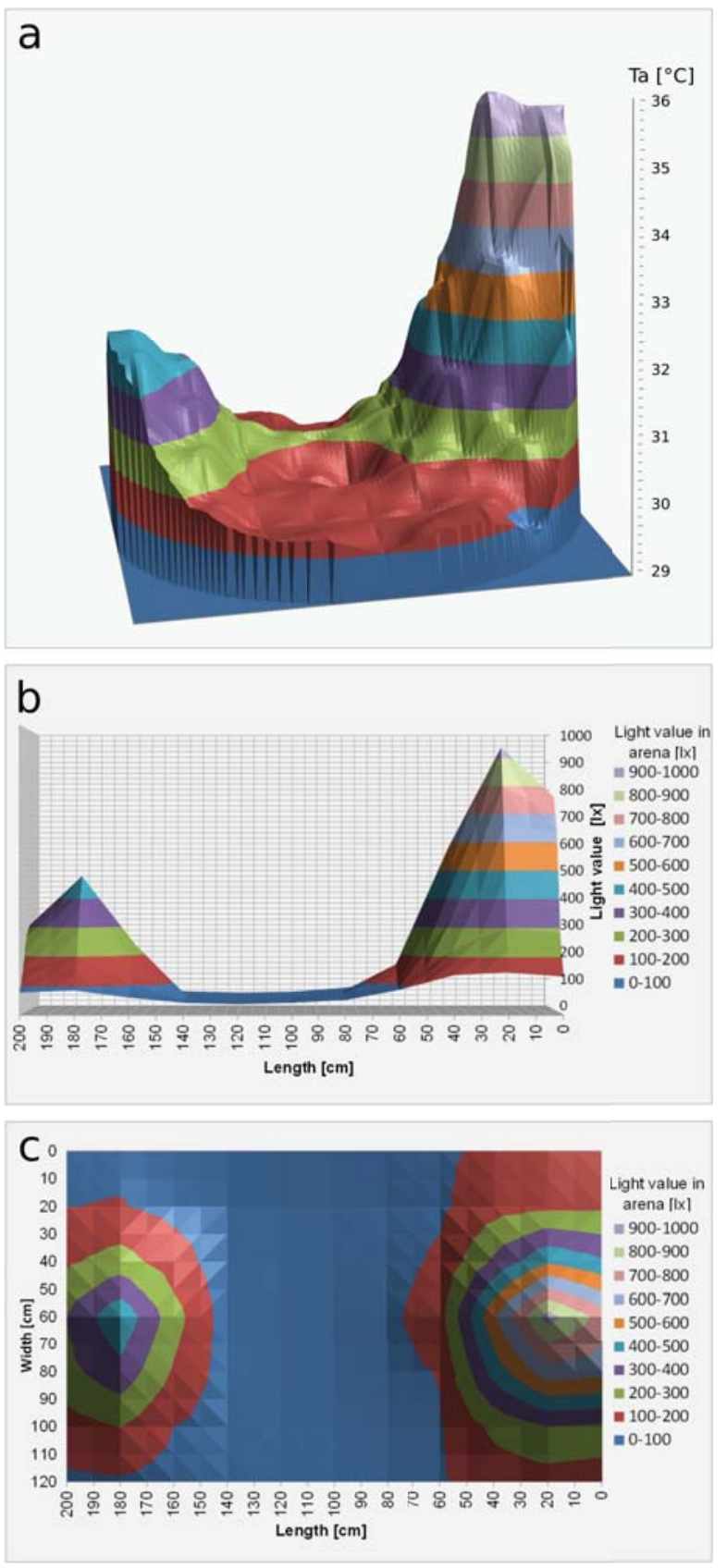

Figure 5: Maps of the optima (global and local) in a luminance field. (a) 3-dimensional representation of the different temperatures within the arena of the honeybee experiments. The targeted temperatures were $36^{\circ} \mathrm{C}$ at the global optimum (right side), $32^{\circ} \mathrm{C}$ at the local optimum (left side) and $31^{\circ} \mathrm{C}$ at the pessimum (center of the arena). (b) Luminance values $[\mathrm{lx}]$ within the arena for robotic experiments as seen from the side. The targeted luminance values were $1000 \mathrm{~lx}$ at the global optimum (right side of the arena), $400 \mathrm{~lx}$ at the local optimum (left side of the arena) and $0 \mathrm{~lx}$ at the pessimum (center of the arena). (c) Luminance values within the arena for robotic experiments as seen from above.

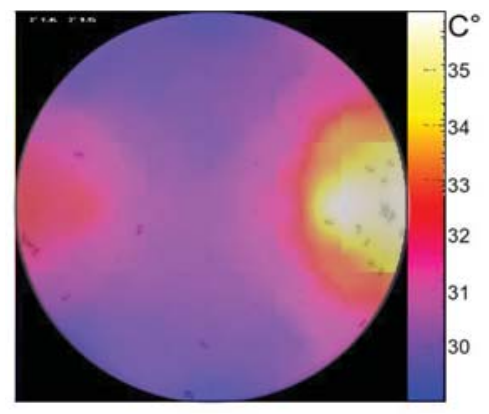

Figure 7: Arena of real-life honeybee experiments with color-coded representation of the temperature gradient within the arena. Targeted temperatures: optimum (right zone) $36^{\circ} \mathrm{C}$, center of the arena (outside the optima) $31^{\circ} \mathrm{C}$ and local optimum (left zone) $32^{\circ} \mathrm{C}$.

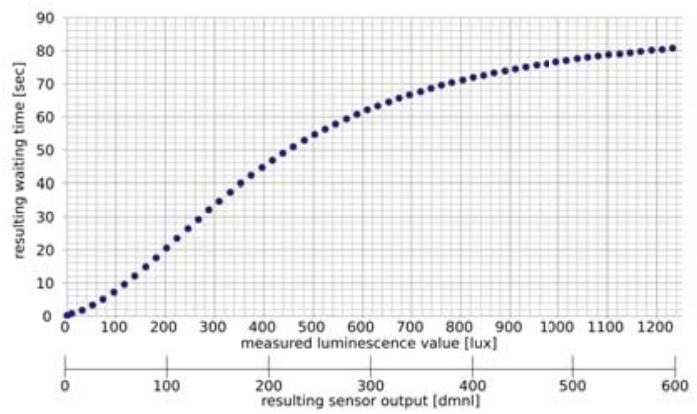

Figure 8: Graph of the fuction Eq. 1 using the values $u=0.00225$ and $m=0.000023$. Dependency of the waiting time duration on the light intensity as measured by the robot (lower $\mathrm{x}$-axis label), as well as the actual light intensity (upper x-axis label). Modified version of the waiting time function, inspired by [22]. Correlation between sensed luminance values of the robots and actual luminance values is shown in Fig. 3.

\subsection{Analyses}

\subsection{Implementation of BEECLUST on robots}

We used e-puck robots (see Fig. 4) as a hardware platform [2] for our experiments. These robots have a round base with a diameter of $7 \mathrm{~cm}$ and are equipped with 8 infrared sensors (Fig. 4c) capable of measuring ambient light and the proximity of objects, used here for obstacle avoidance as well as the detection of other robots. As we used light for creating a complex environment with a global and a local optimum we designed an extension board capable of measuring luminance values. The e-puck robots in our experiments were adjusted to a mean velocity $15 \mathrm{~cm} / \mathrm{sec}$. In comparison to honeybees (the mean crawling speed of bees is about 1.5 body lengths per second), our robots have a slightly higher velocity (around 2 robot lengths per second). The higher velocity of the robots leads to a reduced run time in robotic experiments. All robots were initially placed randomly into the area outside the optima, their initial heading 


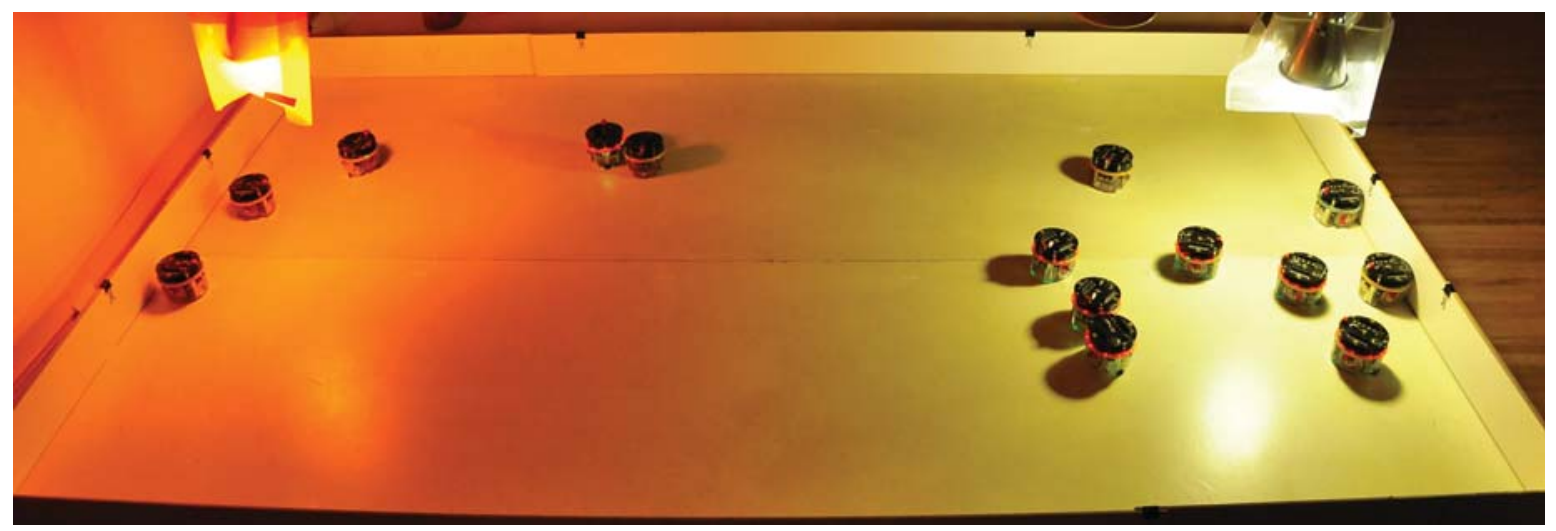

Figure 6: Setup of the arena running a robotic experiment. Left side: Local optimum with a dimmed light source. Right side: Global optimum, light source is also covered in plastic sheets to replicate possible distortion.

was randomized $\left(0^{\circ}<\right.$ heading $\left.<360^{\circ}\right)$.

One of the most important properties of the BEECLUST algorithm is the translation of the environmental parameter in focus (e.g. temperature, light intensities...) to a waiting time after each agent-agent contact. Here we used a modified version of a waiting time function proposed by [22], Eq. 1. The waiting time function is shown in Fig. 8, plotted against the light intensity perceived by the robots as well as the actual light intensity.

$$
w(t)=\left\{\begin{aligned}
\frac{m \cdot s(t)^{3}}{u \cdot s(t)^{3}+s(t)} & \text { if } s(t)>0 \\
0 & \text { otherwise }
\end{aligned}\right.
$$

The waiting-time variable $w(t)$ depicts the waiting-time of each robot in seconds as a result of Eq. 1. The variable $s(t)$ stands for the sensed luminance value of the TCS3200 light sensor. The parameter $u$ controls the steepness of the waiting-time function and the parameter $m$ scales the waiting time function on the y-axis. For the experiments reported here, we used the values of $u=0.00225$ and $m=$ 0.000023 , resulting in a waiting-time function seen in Fig. 8.

\subsection{Results}

Fig. 9 shows the distribution of honeybees and robots in their respective experiments. Fig 9a shows that most of the honeybees (median of $70 \%$ ) were located at the global optimum. This is significantly more than the Uniform random Distribution Model (UDM) hypothesizes (for the global optimum: $11.2 \%$ ). The second most bees were located in the pessimum (median of $15.6 \%$ ), distinctly less than predicted by the UDM $(77.6 \%)$. The fewest bees were located in the local optimum (median of $12.3 \%$ ), which is very close to what the UDM predicts (11.2\%). Fig 9b shows a very similar distribution for the robotic experiments. Most robots were located at the global optimum (median of $55 \%$, UDM: $11.2 \%$ ), the second most at the pessimum (median of $25 \%$, UDM: $77.6 \%$ ) and the fewest at the local optimum (median of $15 \%$, UDM: $11.2 \%$ ).

In Fig. 10 the results of all experiments with different number of social seed agents are shown. Fig. 10a shows that with no social seed agent in the global optimum around $55 \%$ (median) of the robots are located in the end of the experi- ments. The second most robots were located in the experiments with one social seed agents (median of $35 \%$ ), followed by the experiments with two social seed agents (median of $30 \%$ ). All results are distinctly above the predicted values of the UDM. Fig. 10b shows that we found a very similar percentage of robots over all experiments at the area outside the optima (pessimum). At the end of the runs, the fewest robots were located here using no social seed agent (median of $25 \%$ ), followed by experiments with one social seed agent (median of $30 \%$ ) and experiments with two active social seed agents (median of 30\%). The percentage of robots outside the optima is considerably lower than the UDM hypothesized for it. Fig. 10c shows the percentage of robots at the local optimum. While the fewest robots gathered here in experiments with no social seed agent (median of $15 \%$ ), one social seed agent led to a significant increase of robots in this area (median of 20\%). Two social seed agents led to even more robots gathering in the local optimum (median of $35 \%)$.

In Fig. 11 we show that different numbers of social seed agents lead to a different distribution of waiting time activity in both optima. The percentage of the median number of robots in the global (red dots) and the local optimum (blue dots) for different numbers of social seed agents is shown. In Fig. 11a we additionally show the median of the percentage of real honeybees over the course of the experimental time (global optimum: yellow squares, connected via an interpolated line (red), local optimum: light blue squares, connected via an interpolated line (light blue)). As seen in comparison to the robotic experiment results, the difference between the temporal distribution between global and local optima decreases with an increasing number of social agents. In the temporal distributions of Fig. 11b (using one social seed agent) and Fig. 11c, the effect of social seed agents can be seen. In experiments with one social seed agent, the difference between the number of robots in the global and the local optima diminishes, which is shown even more prominently in experiments using two social seed agents.

\subsection{Discussion}

Our study showed that the BEECLUST algorithm was implemented successfully on a swarm of e-puck robots, and we showed in detail how this re-embodiment of honeybee 


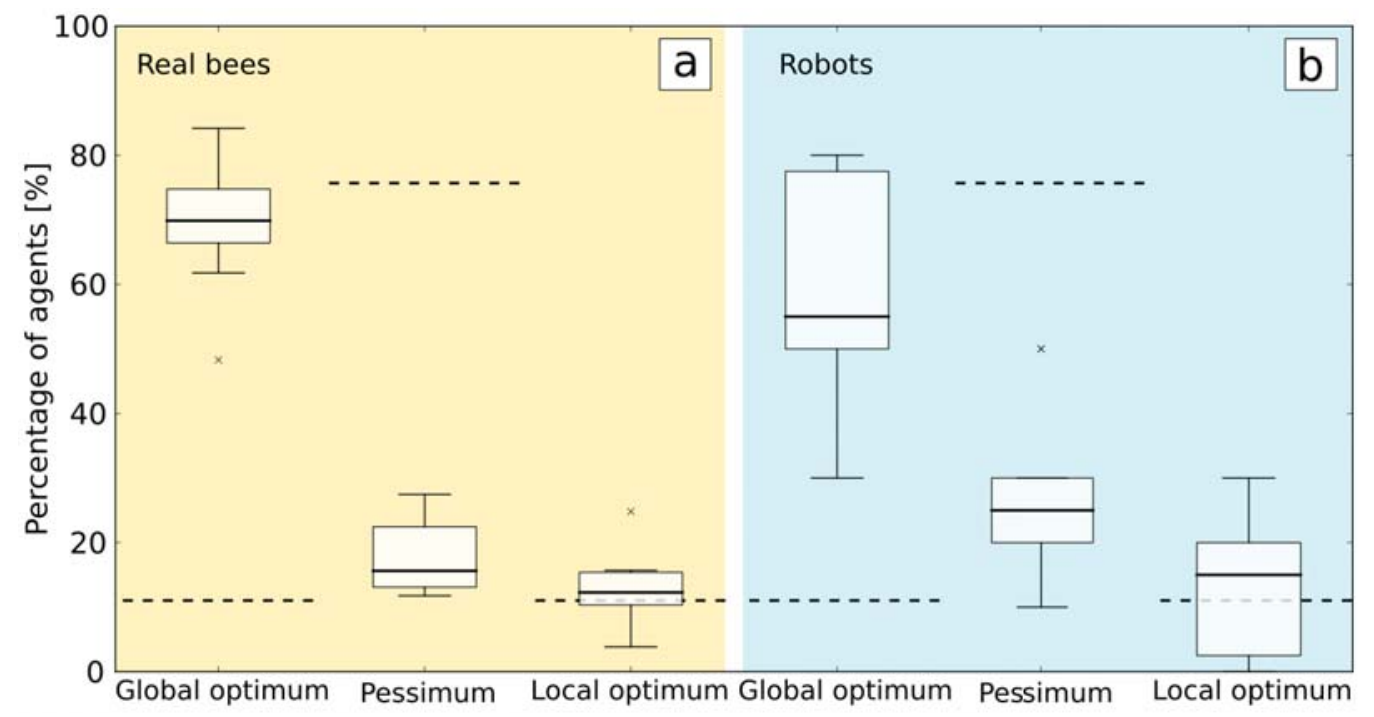

Figure 9: Results of honeybee experiments in comparison with robot experiments. (a) Median percentage of bees in the three different optima zones: global optimum, $36^{\circ} \mathrm{C}$, local optimum (suboptimum), $32^{\circ} \mathrm{C}$ and outside the optima (pessimum), $31^{\circ} \mathrm{C}$. Pooled results of experiments with honeybees (groups of 6, 24, 64 and 128 with $\mathrm{N}=8$ runs for each group size) after 30 minutes of run time. (b) Median percentage of robots in the three different optima zones (global optimum, $1000 \mathrm{~lx}$; local optimum, $400 \mathrm{~lx}$ and outside the optima (pessimum), $0 \mathrm{~lx}$ ). 10 robots per run were used with $\mathrm{N}=10$ repetitions and a run time of 10 minutes. The dashed line represents the predicted values by the Uniform random Distribution Model, deviations from this line indicate that agents locations are not chosen independently.

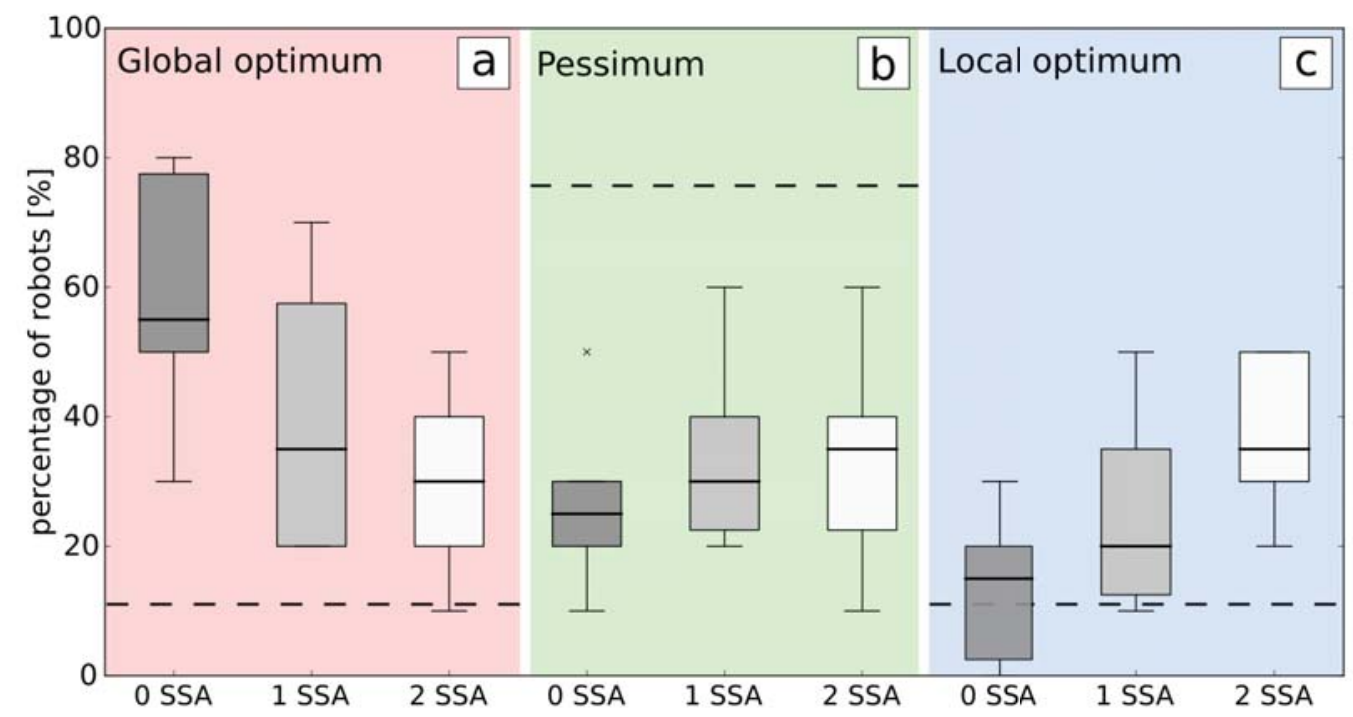

Figure 10: Percentage of robots for the three tested number of social seed agents (dark gray filled boxplots: no social seed agent, light gray filled boxplots: one active social seed agent, white filled boxplots: two social seed agents). (a) Robots at the global optimum at $1000 \mathrm{~lx}$. (b) Robots at the local optimum, $400 \mathrm{~lx}$. (c) Robots outside the optima (pessimum), $0 \mathrm{~lx} . \mathrm{N}=10$ repetitions/number of social seed agents. The dashed line indicates the predicted values by the Uniform random Distribution Model for these zones. The dashed line represents the predicted values by the Uniform random Distribution Model. Deviations from this line indicate that agents locations are not chosen independently. 


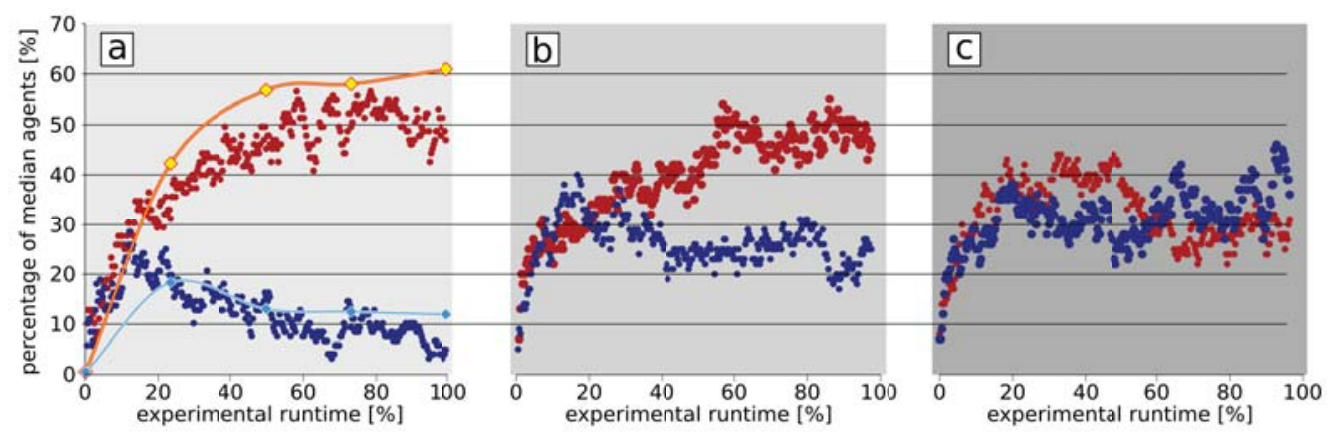

Figure 11: Median number of agents at the global and local optimum normalized according to the total number of participating agents, hence the percentage $(\mathrm{N}=10)$. The number of robots in active waiting mode was counted for every two seconds. The percentage of the median number of robots in the global (red dots) and the local optimum (blue dots) for different numbers of social seed agents is shown for: (a) no social seed agent, (b) one social seed agent and (c) two social seed agents. In (a) we additionally show the percentage of the median number of real honeybees over the course of the experimental time (pooled data, global optimum: yellow squares, connected via an interpolated line (red), local optimum: light blue squares, connected via an interpolated line (light blue)).

behaviors was performed: We had to translate the honeybee behavior (walking in temperature gradient fields) into a swarm robotic implementation on e-puck hardware operating in luminance fields (light gradient fields). We tested several swarm configurations in a binary choice setting with a global optimum on the right side and a local optimum on the left side. This experimental setting is a classical setup in experimenting the swarm intelligence in social insects, e.g. the "double bridge experiment" in ants [7], the "feeder-choice setup" in honeybees [23] or the "two-shelter setup" in cockroaches [3]. The shelter choice was successfully re-embodied into swarm robots and already [11] used in mixed societies of cockroaches and cockroach-like robots [12]. A similar approach is pursued by us in the project ASSISIbf [1], with the aim to extract behavior of honeybees and fish and the re-embody them into robots in order to generate mixed societies with their natural counterparts. The ability of the swarm to discriminate between an global optimum and a local optimum was first demonstrated (Fig. 9b) and compared to honeybee behavior in luminance gradient fields (Fig. 9a). Then we demonstrate that a "social seed robot" in the local optimum can draw a fraction of the swarm to the other side (Fig. 10a, b, c) and that two social seed robots can attract an even greater proportion to the other side. The observed dynamics of swarms without social seed in this decision making are showing patterns over time which are comparable to those found in honeybees (11). Analysis showed that the convergence to a global optimum takes on average $8 \mathrm{~min}$ utes ( $80 \%$ of the experimental run time), while the final system state is reached with two social seeds already after 2 minutes (20\% of the experimental run time). Thus the effect of the social seed is assumed to be rather "cutting off" the further convergence to the global optimum side than lowering the carrying capacity of any given side. The temporal distribution of robots using no social seed agent (Fig. 11) also indicates competitive inhibition: For the first 2 minutes the number of robots in both optima increases $(20 \%$ of the experimental run time). After that only the number of robots in the global optimum increases further, reducing the number of robots in the local optimum. Social seed agents subdue this effect, as can be seen in Fig. 11b and 11c.

The main implication of our finding is that a swarm driven by simple social interaction algorithms (e.g. BEECLUST) can be easily affected, controlled or modulated by adding new interaction-patterns, like our social seed robots. They interact normally with their swarm mates, however they just insist in locating themselves around the local optimum. This shows that even an already existing swarm, maybe one that is already running somewhere, can be post-hoc reprogrammed by introducing socially modulating agents. This is significant for future swarm robotics and swarm intelligence research but also clearly indicates a potential security problem: An intended swarm behavior is prone to such attacks, especially because no access to the internal interconnection of individual agents is required. There are other more common swarm systems running with rather "fixed interaction programs" since a long time: animal societies, human society, markets and economies, traffic networks and social networks. In all these systems agents interact with simple stimulus-response-triggered behaviors which are sometimes modulated by learning (short term) or evolution (long term). Introducing special agents offers a way to get control over these systems "from the inside" without the need to reprogram them, what is not possible in most cases. This offers the door for many future applications: Robots that mimic natural organisms to gain control over these natural swarm systems $[1,9]$, chatbots in social media or even "benevolent agents-provocateur cars" which have the only purpose to interact with other cars in a way that they draw, in a subtle way, parts of the self-organizing traffic flow to other roads, this way bringing relief to the overall system.

\section{AUTHOR CONTRIBUTION}

M.St. conducted all the experiments and made numerical analysis. M.Sz. provided honeybee data for comparison. R.T. helped with python coding, statistics in data analysis for robots and supervised the experimental design. G.R. 
provided the necessary computerized tracking of agents. T.S had the initial BEECLUST idea, the initial experimental setup design, and the initial code. He designed the study and the article and provided the e-puck swarm.

\section{ACKNOWLEDGEMENTS}

Supported by EU FET project ASSISIbf, \#601074; Austrian Science Fund (FWF) grant P23943-N13 (Rebodiment).

\section{REFERENCES}

[1] ASSISI|bf. Animal and robot societies self-organise and integrate by social interaction (bees and fish). http://assisi-project.eu. project website, 2015.

[2] ePuck e-puck desktop mobile robot. http://www.e-puck.org/. Accessed: 2016-09-30.

[3] J.-M. Ame, C. Rivault, and J.-L. Deneubourg. Cockroach aggregation based on strain odour recognition. Animal Behaviour, 68:793-801, 2004.

[4] E. Ben-Jacob. Learning from bacteria about natural information processing. Annals of the New York Academy of Sciences, 1178(1):78-90, 2009.

[5] E. Bonabeau and C. Meyer. Swarm intelligence: A whole new way to think about business. Harvard Business review, 79(5):106-115, 2001.

[6] C. Detrain, J.-L. Deneubourg, and J. M. Pasteels. Decision-making in foraging by social insects. In Information Processing in Social Insects, pages 331-354. Springer, 1999.

[7] A. Dussutour, V. Fourcassié, D. Helbing, and J.-L. Deneubourg. Optimal traffic organization in ants under crowded condition. Nature, 428:70-73, 2006.

[8] J. R. Dyer, A. Johansson, D. Helbing, I. D. Couzin, and J. Krause. Leadership, consensus decision making and collective behaviour in humans. Philosophical Transactions of the Royal Society of London B: Biological Sciences, 364(1518):781-789, 2009.

[9] flora robotica. http://www.florarobotica.eu/. project website, 2016.

[10] S. Garnier, J. Gautrais, and G. Theraulaz. The biological principles of swarm intelligence. Swarm Intelligence, 1(1):3-31, 2007.

[11] S. Garnier, C. Jost, R. Jeanson, J. Gautrais, M. Asadpour, G. Caprari, and G. Theraulaz. Aggregation behaviour as a source of collective decision in a group of cockroach-like-robots. In M. Capcarrere, editor, Advances in artificial life: 8th European conference, ECAL 2005, volume 3630 of LNAI, pages 169-178. Springer-Verlag, September 2005.

[12] J. Halloy, G. Sempo, G. Caprari, C. Rivault, M. Asadpour, F. Tâche, I. Saïd, V. Durier, S. Canonge, J. M. Amé, C. Detrain, N. Correll, A. Martinoli, F. Mondada, R. Siegwart, and J. L. Deneubourg. Social integration of robots into groups of cockroaches to control self-organized choices. Science, 318(5853):1155-1158, November 2007.

[13] D. Kengyel, R. Thenius, K. Crailsheim, and T. Schmickl. Influence of a social gradient on a swarm of agents controlled by the beeclust algorithm. In European Conference on Artificial Life, pages 1041-1048, 2013.
[14] D. Kengyel, P. Zahadat, T. Kunzfeld, and T. Schmickl. Collective decision making in a swarm of robots: How robust the beeclust algorithm performs in various conditions. In Proceedings of the 9th EAI International Conference on Bio-inspired Information and Communications Technologies (Formerly BIONETICS), BICT'15, pages 264-271, 2016.

[15] S. Kernbach, R. Thenius, O. Kernbach, and T. Schmickl. Re-embodiment of honeybee aggregation behavior in an artificial micro-robotic system. Adaptive Behavior, 17(3):237-259, 2009.

[16] E. Mallon, S. Pratt, and N. Franks. Individual and collective decision-making during nest site selection by the ant leptothorax albipennis. Behavioral Ecology and Sociobiology, 50(4):352-359, 2001.

[17] P. Michelena, R. Jeanson, J.-L. Deneubourg, and A. M. Sibbald. Personality and collective decision-making in foraging herbivores. Proceedings of the Royal Society of London B: Biological Sciences, 277(1684):1093-1099, 2010.

[18] M. Nagy, Z. Akos, D. Biro, and T. Vicsek. Hierarchical group dynamics in pigeon flocks. Nature, 464(7290):890-893, 2010.

[19] R. Popat, D. Cornforth, L. McNally, and S. Brown. Collective sensing and collective responses in quorum-sensing bacteria. Journal of the Royal Society Interface, 12(103):20140882, 2015.

[20] H. Schau, A. Hemetsberger, and R. Kozinets. The wisdom of consumer crowds. Journal of Macromarketing, 28(4):339-354, 2008.

[21] T. Schmickl and H. Hamann. Beeclust: A swarm algorithm derived from honeybees. Bio-inspired Computing and Communication Networks. CRC Press (March 2011), 2011.

[22] T. Schmickl, R. Thenius, C. Moeslinger, G. Radspieler, S. Kernbach, M. Szymanski, and K. Crailsheim. Get in touch: cooperative decision making based on robot-to-robot collisions. Autonomous Agents and Multi-Agent Systems, 18(1):133-155, 2009.

[23] T. D. Seeley, S. Camazine, and J. Sneyd. Collective decision-making in honey bees: how colonies choose among nectar sources. Behavioral Ecology and Sociobiology, 28(4):277-290, 1991.

[24] D. J. Sumpter, J. Krause, R. James, I. D. Couzin, and A. J. Ward. Consensus decision making by fish. Current Biology, 18(22):1773-1777, 2008.

[25] M. Szopek, T. Schmickl, R. Thenius, G. Radspieler, and K. Crailsheim. Dynamics of collective decision making of honeybees in complex temperature fields. PloS one, 8(10):e76250, 2013.

[26] M. Vuković. Crowdsourcing for Enterprises. In Services - I, 2009 World Conference on, volume 0, pages 686-692, Los Alamitos, CA, USA, July 2009. IEEE. 